\title{
Spontaneous acne fulminans treated with corticotherapy, antibiotics and oral isotretinoine
}

\begin{abstract}
This pathology is a rare and serious form of acne vulgaris. Its treatment is a challenge because it does not respond satisfactorily to traditional acne therapies. Its diagnosis must be early so that the appropriate therapy is instituted quickly, avoiding sequelae. The case reported is a young male patient with acneic lesions associated with systemic manifestations and laboratory abnormalities. Treatment with prednisone and oral antibiotic therapy is instituted. After weaning from corticosteroid therapy, oral isotretinoin was introduced in a stepwise dose. The patient progresses with improvement of active lesions and cicatricial lesions on the back. The Acne Fulminans is destructive, begins with acute pain, with abrupt development of acneic lesions, hemorrhagic nodules and ulcerations with necrotic background, associated with systemic manifestations. The diagnosis is clinical. Therapy should be aggressive, involving oral corticosteroids with isotretinoin. Dapsone is also used as an antiinflammatory agent.
\end{abstract}

\author{
Volume 2 Issue 5 - 2018
}

\section{Denise Camilios Cossiolo, Ana Cecília Siqueira Camargo, Maria Fernanda Camargo Boin}

Department of Medicine, Catholic University of Paraná, Brazil

Correspondence: Denise Camilios Cossiolo, Medicine Student, Catholic University of Paraná,Av. Jockey Club, 485 -Hipica, Londrina-PR, Brazil, Tel 43999462733 ,

Email deniseccossiolo@gmail.com

Received: August 20, 20I8 | Published: September 20, 2018

\section{Introduction}

The Acne Fulminans (AF) was first described in 1959 by Burns and Colville. ${ }^{1,2}$ Subsequently, Kelly and Burns (1971) introduced the term "Acute febrile ulcerative acne conglobata with polyarthralgia". Plewig and Kligman, in 1975, began to use the term acne fulminans, separating it from acne conglobata, emphasizing the sudden onset and severity of the disease. ${ }^{3}$ This pathology consists of a rare and severe form of acne vulgaris associated with systemic symptoms. It mainly affects adolescent males. ${ }^{1}$ Its etiology is unknown, but there is evidence of being multifactorial. ${ }^{4,5}$ They associate this pathology to the increase of androgens, to immunological causes and to the genetic predisposition..$^{1,3}$ Their diagnosis is usually clinical. ${ }^{5}$ The treatment of Acne Fulminans is a challenge, since the response to traditional acne therapies is not satisfactory. Therefore, it is recommended an aggressive therapy composed of an association of oral corticosteroids and isotretinoin. ${ }^{1}$ Although the prognosis after therapy is satisfactory, the skin lesions may leave scars and milia. ${ }^{2}$

\section{Case report}

A male patient, 13years old, with a history of acne lesions on the face and trunk for two months with reports of fever, myalgia, weight loss and laboratory abnormalities of transaminases and leukocytosis. Patient presented to the physical examination lesions characterized by ulcers covered by hemorrhagic crusts. He had used Lymecycline (300mg, 1x/day) during one month without therapeutic response. Initial treatment with prednisone $1 \mathrm{mg} / \mathrm{kg}$ and oral antibiotic therapy with Amoxicillin Clavulanate for twenty-one days. After starting weaning from corticosteroids and introducing oral Isotretinoin in a step dose of $10 \mathrm{mg}$ to $40 \mathrm{mg} / \mathrm{day}$, with a total dose of $120 \mathrm{mg} / \mathrm{kg}$. Patient progresses with improvement of active lesions and cicatricial lesions on the back (Figure 1) (Figure 2).

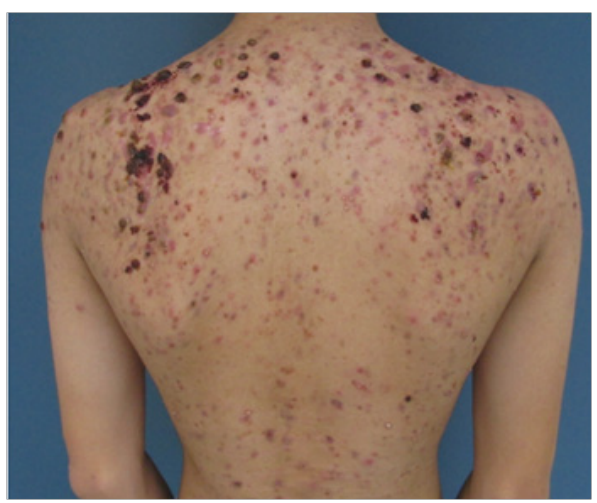

Figure I Before treatment for Acne Fulminans.

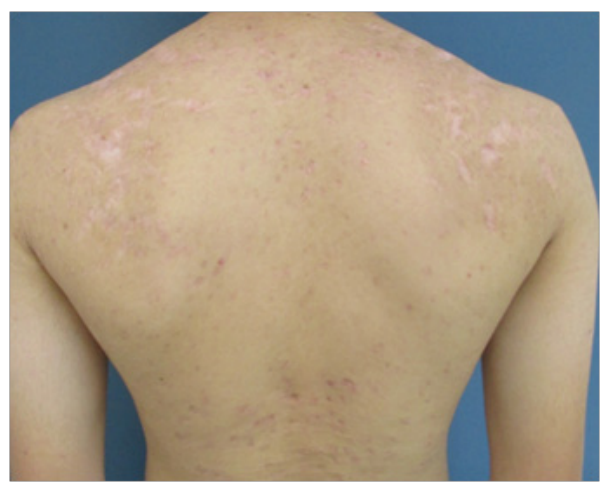

Figure 2 After treatment for Acne Fulminans (corticosteroid therapy+isotretinoin at step dose). 


\section{Discussion}

\section{Physiopathology}

The pathogenesis of AF is not fully understood, but heredity, exacerbated immune reactions, bacterial infections and the use of some medications, as well as: Isotretinoin, Tetracycline and Testosterone, can be triggering factors. ${ }^{4,6}$ There is also a hypothesis that there is a deregulation of some receptors expressed by the sebaceous glands and keratinocytes, in particular, the androgen receptors. The possible association of genetic factors in the etiopathogenesis of acne is based on the finding that a family history of acne represents a greater risk for the development of this pathology.?

The association of AF with the use of oral isotretinoin is probably due to an exacerbated hypersensitivity reaction type III and IV, after a massive contact with Propionibacterium acnes antigens favored by the fragility of the pilosebaceous epithelium, induced by this drug. Another theory is a change in the function of neutrophils, genetically determined, leading to hyper reactivity to inflammatory mediators and a decrease in phagocytosis of $P$. acnes that, in a high proportion, would explain the development of AF at the beginning of oral Isotretinoin therapy. ${ }^{4}$ The doses and the intervals between the medication and the acute manifestations vary. However, lesions generally appear between the fourth and eighth week of the beginning of treatment. Some authors affirm that the occurrence of the lesions is dose dependent, others do not. The AF linked to the use of isotretinoin needs to be differentiated from acne tetrad and pseudoacne fulminans. Acne tetrad consists of acne conglobata, suppurative hydroadenitis, pilonidal cyst and folliculitis abscess. ${ }^{2}$ The pseudoacne fulminans is manifested by sudden worsening of acne without systemic symptoms and laboratory abnormalities. ${ }^{4}$ This is due to the capacity of the drug to determine changes in desmosomes, collagen and vascular proliferation. ${ }^{2}$ High blood levels of testosterone may also can initiate an important role in the pathogenesis of AF, this explains why this disease affects, in the great majority, male adolescents. ${ }^{2,3}$ In addition, the literature has described cases of exacerbation of the pathology during therapy with testosterone. ${ }^{3,7}$ These cases are linked to two situations: selfmedication with anabolic steroids in bodybuilders or testosterone treatment for individuals of high stature. Of the cases cited by Saint et al. ${ }^{7}$ five developed AF between 3 and 18months after the start of treatment with testosterone and one after the end of treatment. This delay may be due to the time to reach a serum testosterone level capable of activating the androgen receptors present in the skin. ${ }^{7}$

\section{Epidemiology}

The AF is mainly present in Caucasian male adolescents, 13 to 22 years old, with mild to moderate acne. ${ }^{2,3,6}$ It is estimated to occur in less than $1 \%$ of all cases of acne. ${ }^{2}$ The incidence of AF is decreasing, probably due to the adequate and early treatment of both mild and moderate acne. $^{8}$

\section{Clinical manifestations}

Acne Fulminans is destructive; it starts with acute pain, with abrupt development of multiple papules, plaques, hemorrhagic nodules and ulcerations with necrotic background. They affect the face, the thoracic region, the back, arms and rarely the thigh. ${ }^{1,6,8}$ Usually systemic manifestations are present at the beginning and are: fever, asthenia, chills, weight loss, musculoskeletal pain, arthralgia, myalgias and osteolytic lesion. ${ }^{1,2,4,6,9}$ Due to the severity of the systemic symptoms, the patients may require rest and hospitalization. ${ }^{1}$ The most affected joints are: iliossacral, ankles, shoulders and knees. Polyarthritis can cause pain when moving, so patients tend to assume an arched posture. ${ }^{3}$ The predominant sites of bone lesions are clavicle, sternum, and ends of long bones. It is proposed that in the bones, as well as in the skin, certain antigens found have similarity to bacterial antigens, triggering an immune reaction of hypersensitivity responsible for the bone lesions. ${ }^{6}$ In the literature has described cases related to Crohn's disease and after measles infection. ${ }^{6}$ There are reports of erythema nodosum and hepatosplenomegaly associated with AF, but they are more rare. ${ }^{8}$ Patients also present laboratory abnormalities such as leukocytosis with neutrophilia, increased erythrocyte sedimentation rate, thrombocytosis, microscopic hematuria, elevation of transaminases, ESR and PCR1. 2,6,9 The blood cultures of patients with $\mathrm{AF}$, mostly, do not show bacterial growth, excluding the possibility of being a septic picture. In skin cultures, there is a predominance of Staphylococcus epidermidis and Propionibacterium acnes and, rarely, of Pseudomonas, Staphylococcus aureus and Streptococcus pyogenes. $^{2}$

\section{Diagnosis}

The diagnosis of AF is usually clinical and presents as differential diagnoses pseudoacne fulminans, acne conglobata, rosacea fulminans and SAPHO syndrome (synovitis, acne, pustulosis-usually palmoplantar, hyperostosis, osteitis). ${ }^{3,5,9,10}$ On the other hand, acne conglobata is found in sites similar to AF, located mainly in the trunk and upper limbs and rarely in the face. However, it occurs in older individuals, has a more chronic course without abrupt onset, have inflammatory cystic lesions and multiple comedones, besides not presenting systemic symptoms. While rosacea fulminans has a sudden onset, it often affects adult women, after a period of stress and without a previous history of acne. The systemic symptoms are non-existent, lesions are located in the central region of the face and comedones are rare. ${ }^{3}$ Regarding SAPHO it is believed that Propionibacterium acnes cause an autoimmune chronic inflammatory process in individuals with genetic predisposition. ${ }^{10}$ Its diagnosis should be considered when coexisting inflammatory bone, recurrent, dermatoses and arthritis, especially in the absence of autoantibodies and infectious agents, in addition to the persistence of laboratory markers of inflammation. ${ }^{11}$

Burns and Colville proposed five criteria for the diagnosis of AF. They are:
i. Sudden onset of the lesions
ii. Severe ulcerated acne that causes scarring
iii. Systemic symptoms such as fever and polyarthralgia
iv. Absence of response to antibiotics
v. Favorable response to corticotherapy. ${ }^{4}$

In addition, Karvonen defined criteria for the clinical and laboratory diagnosis of AF. There are absolutes (severe ulcerated nodules, acute onset acne, arthralgia and/or myalgia after exercisefor at least a week) and the relative (fever $>38^{\circ} \mathrm{C}$ for at least one week, leukocytosis $>10000 / \mathrm{mm}^{3}, \quad$ ESR $>50 \mathrm{~mm} / \mathrm{h}, \quad \mathrm{PCR}>50 \mathrm{mg} \%$ and osteolytic lesions on radiographs, bone scans or tomography). The diagnosis is made in the presence of two absolute and two relative criteria. ${ }^{8}$ Bone scintigraphy can be used for the detection of inflammatory and lytic lesions which cause an increase in the uptake of the radioindicator. ${ }^{6}$ The histopathological changes present in AF are extensive areas of skin necrosis, with ulcerations covered by 
parakeratotic crusts formed by polymorpho nuclear clusters.

The ulcer base is covered by necrosis, fibrinoid detritus and edema. The inflammatory infiltrate is composed of polymorph nuclear cells intact and lysed, incorporated into partially destroyed vessels and apocrine sweat glands. ${ }^{8}$ Studies involving immunohistochemistry of acne lesions, including AF, are rare in the literature. The CD20 is considered a membrane antigen, B lymphocytes marker. Only in severe forms of acne there is inflammatory infiltration with CD20 + cells. Anti-CD3 antibody reacts to the CD3 antigen present in T cells. CD3 + cells were found in all cases of acne, emphasizing the intervention of cellular immunity in triggering the inflammatory response in acne, by means of type IV hypersensitivity reaction8. The CD68 consists of a $110-\mathrm{Kd}$ glycoprotein bound to lysosomal granules, responsible for the marking of monocyte/macrophage lineage. ${ }^{4,12}$ In acne lesions there are many CD68 + cells represented by macrophages, epithelioid cells and giant cells, associated with $\mathrm{T}$ lymphocytes and polymorphonuclear. They trigger granulomatous reactions, similar to that of foreign body granuloma. This can be explained by the behavior of comedones content (including sebum) as a "no self" antigen complex, causing a delayed hypersensitivity response (type IV). ${ }^{8}$

\section{Treatment}

The treatment of AF is controversial and there is still no standardization. There is no drug capable of controlling and preventing seizures. The drugs used are effective only when their use is prolonged, providing several side effects. The available medications are: glucorticoids and isotretinoin. Systemic glucorticoids improve considerably skin lesions and systemic symptoms, but relapse occurs when it does is decreased. Thus, they have to be maintained at a high dose for a variable period, for two to four months and then reduced slowly. It was observed that the association with Dapsone ensures better control of relapses. ${ }^{2}$ Dapsone acts as an anti-inflammatory, avoiding lipid peroxidation and neutrophil recruitment. ${ }^{13}$ The administration of isotretinoin should be gradual because of the risk of worsening symptoms. ${ }^{6}$ Its mechanism of action is anti-inflammatory combined with its known sebostatic function and its effect on follicular keratinization pattern. ${ }^{2}$ Although, this drug is a triggering factor it is indicated in the treatment of acne fulminans at low initial doses and subsequent increase obeying the tolerance of the patient Systemic antibiotics do not alter the course of the disease, but may be associated for the control of secondary infections. ${ }^{2,6}$ In addition, it needs to establish local care of the lesions with compresses and emollients. ${ }^{6}$

Some authors based on its experiences claim that the most effective treatment for this type of acne is the combination of corticosteroids with isotretinoin. According to Seujeran and Cunliffe, the preferred treatment for $\mathrm{AF}$ is oral prednisolone $0.5-1 \mathrm{mg} / \mathrm{kg} / \mathrm{day}$ for $4-6 w e e k s$, associating isotretinoin in the fourth week, initially $0.5 \mathrm{mg} / \mathrm{kg} / \mathrm{day}$, raising the dose gradually. They affirm that this protocol promotes a faster control of the systemic manifestations, besides the elimination of the acne. ${ }^{3}$ However, Giavedoni et al. described a case in which the patient did not respond to treatment with corticosteroid and isotretinoin, being treated successfully from the combination of oral cyclosporine and isotretinoin. ${ }^{5}$ The control of acne and performance of conservative physiotherapy are able to fully resolve musculoskeletal complaints. ${ }^{14}$ Although the prognosis is good for properly treated patients, these acute inflammatory nodules leave residual scars after healing. ${ }^{1}$

\section{Conclusion}

The Acne fulminant is a pathology whose onset is abrupt and whose evolution is rapid. Therefore, it is very important that its diagnosis and its treatment are early, reducing its morbidity. The patient of the cited case did not obtain clinical improvement with the initial treatment, which was directed to acne vulgaris. He only showed improvement when he was given therapy for acne fulminant. Therefore, the correct diagnosis is critical to minimize damage.

\section{Acknowledgements}

None.

\section{Conflict of interest}

Authors declare that there is no conflict of interest.

\section{References}

1. Zara R, Schwartz R, Jarmuda S, et al. Acne fulminans: explosive systemic form of acne. J Eur Acad Dermatol Venereol. 2011;25(5):501-507.

2. Pereira MF, Roncada EM, Oliveira CM, et al. Acne fulminans and isotretinoin: case report. An Bras Dermatol. 2011;86(5):983-985.

3. Lages RB, Bona SH, Silva FV, et al. Acne fulminans successfully treated with prednisone and dapsone. An Bras Dermatol. 2012;87(4):612-614.

4. Grando LR, Leite OG, Cestari TF. Pseudo-acne fulminans associated with oral isotretinoin. An Bras Dermatol. 2014;89(4):657-659.

5. Giavedoni P, Mascaró-Galy JM, Aguilera P, et al. Acne fulminans successfully treated with cyclosporine and isotretinoin. $J$ Am Acad Dermatol. 2014;70(2):e38-e39.

6. Zanelato TP, Gontijo GM, Alves CA, et al. Disabling acne fulminans. An Bras Dermatol. 2011;86(4 Suppl 1):S9-S12.

7. Saint-Jean M, Frenard C, Le Bras M, et al. Testosterone-induced acne fulminans in twins with Kallmann's syndrome. JAAD Case Rep. 2014;18;1(1):27-29.

8. Brănişteanu DE, Cotrutz $\mathrm{CE}$, Luca $\mathrm{MC}$, et al. Morphopathological stigmata in acne fulminans. Rom $J$ Morphol Embryol. 2015;56(3):1185-1190

9. Peleg H, Koslowski B, Hiller N, et al. Radiologic features of acne fulminans. Isr Med Assoc J. 2014;16(6):393-394.

10. Zimmermann P, Curtis N. Synovitis, acne, pustulosis, hyperostosis, and osteitis (SAPHO) syndrome-A challenging diagnosis not to be missed. J Infect. 2016;72 (Suppl):S106-S114.

11. Silva PC, Oliveira FE, Goldenzon VA, et al. Challenges in diagnosis and treatment of a case of SAPHO syndrome in childhood. An Bras Dermatol. 2011;86(4 Suppl 1):S46-S49

12. Falini B, Flenghi L, Pileri S, et al. PG-M1: a new monoclonal antibody directed against a fixative-resistant epitope on the macrophage-restricted form of the CD68 molecule. Am J Pathol. 1993;142(5):1359-7132.

13. Tanghetti EA. The Role of Inflammation in the Pathology of Acne. $J$ Clin Aesthet Dermatol. 2013;6(9):27-35.

14. Lane JM, Leyden JJ, Spiegel RJ. Acne arthralgia. J Bone Joint Surg Am. 1976;58(5):673-675. 